\title{
7374 \\ Clinical Management and Successful Treatment of Bisphosphonates Related Osteonecrosis of the Jaw
}

IMPLANT THERAPY OUTCOMES, SURGICAL ASPECTS
Cesar Luchetti, Alicia Kitrilakis

Department of Implant Dentistry. National University of La Plata, Argentina

\section{Abstract}

Bisphosphonate-related osteonecrosis of the jaw (BRONJ) is a serious oral maxillary or mandibular bone. BRONJ is associated with pain, paresthesia, and oral dysfunction generating an impairment of the quality of life. Treatment of this complication remains difficult. The aim of this study was to develop a conservative and active approach to treat BRONJ. 46 patients over develop a conservative and active approach to treat BRONJ. 46 patients over
last 12 years, who received an active, still conservative treatment, were last 12 years, who received an active, still conservative treatment, were
analyzed. In each case, the protocol was to manipulate the bone exposure by analyzed. In each case, the protocol was to manipulate the bone exposure by
gently trying to loosen it three times a week. The aim is that soft tissue healing begin to occur below the bone exposure before the final removal of the sequestrum. In all cases, after the procedure was done, the soft tissue healed after around one month. No new bone exposure were observed. The latest literature, and also this study, supports the concept that BRONJ may be successfully treated. The approach described here, especially regarding bone exposure management, could minimize necessary surgical corrections upon removal of the sequestrum.

\section{Background and Aim}

Bisphosphonate-related osteonecrosis of the jaw (BRONJ) is a serious oral complication of bisphosphonate treatment involving the exposure of necrotic max lary or mandibular bone. BRONJ is associated with pain, paresthesia, and oral dysfunction generating an impairment of the quality of life. Treatrandicated and the most useful action is prevention. Surgery is normally

The aim of this study was to develop a conservative and active approach to treat BRONJ

\section{Methods and Materials}

46 patients over last 12 years, who received an active, still conservative treatment, were analyzed. In each case, the protocol was to manipulate the bone exposure by gently trying to loosen it three times a week. During each visit, we carefully irrigated and cleansed the area apical to the bony sequestrum using $5 \mathrm{cc}$ of chlorhexidine $0.12 \%$ followed by $5 \mathrm{cc}$ of $3 \%$ hydrogen peroxide. Detritus were eliminated by means of a hand brush. The aim is that soft tissue healing begin to occur below the bone exposure before the final removal of the sequestrum.

Home care included local rinses with chlorhexidine $0.12 \% 3$ times a day and $3 \%$ hydrogen peroxide once a day, and the use of a soft brush to clean the exposed bone.

Antibiotics were used just when an active infection was present. Antibiotics of choice were mainly Ciprofloxacin and Metronidazole, and in some cases Azitromicin, always based in the results of an antibiogram.

After some time, ranging from a few weeks to 4 months, sequestrums were loose enough to attempt removal. We successfully removed the sequestrums with rongeurs and used rotary instruments to eliminate remaining bony spicules to get a smooth bone surface and facilitate healing.

The sequestrums were submitted for histologic examination which revealed necrotic bone with empty lacunaes and associated infected tissue.

Example Case 1: Mandible bilateral exposure
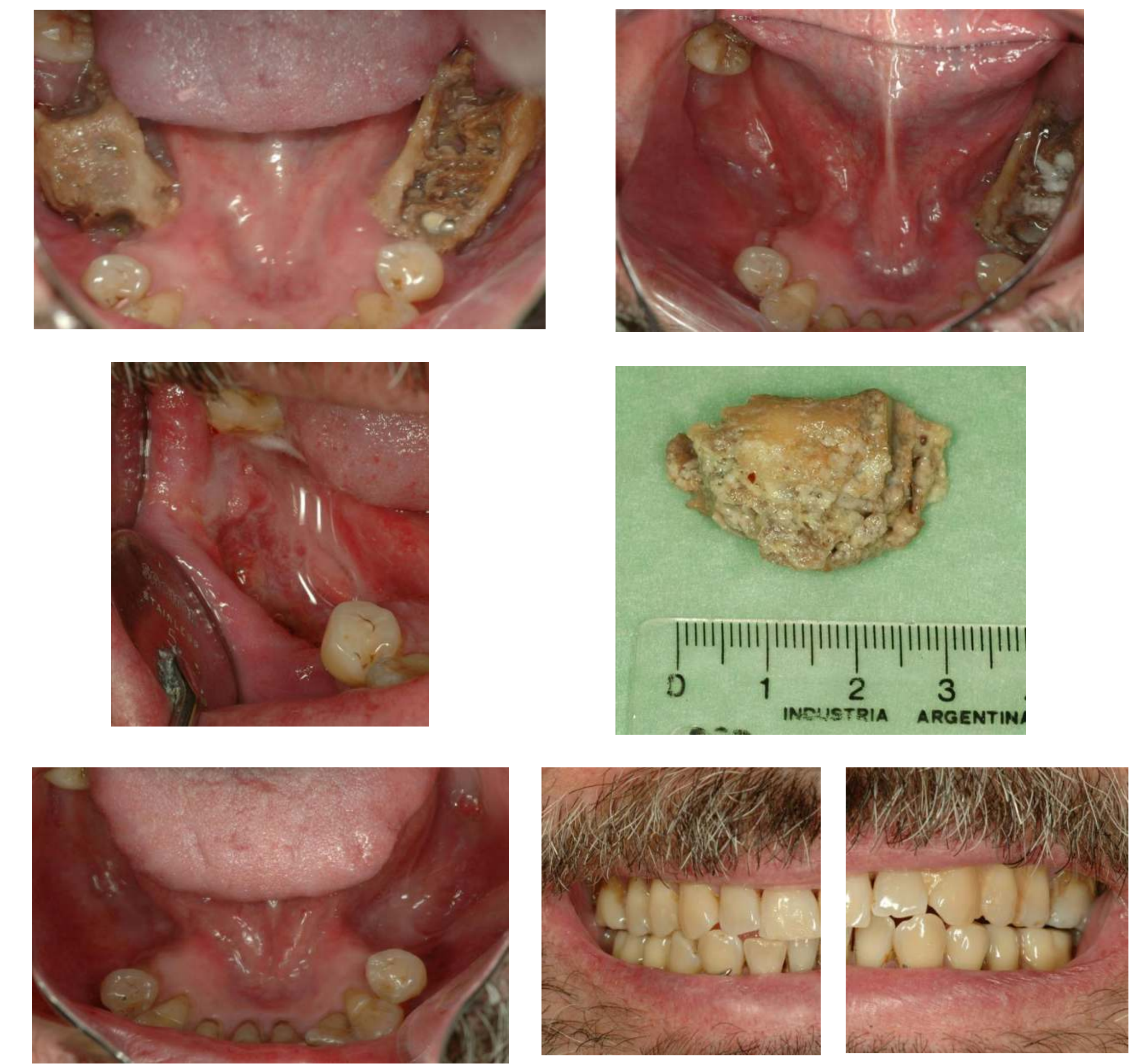
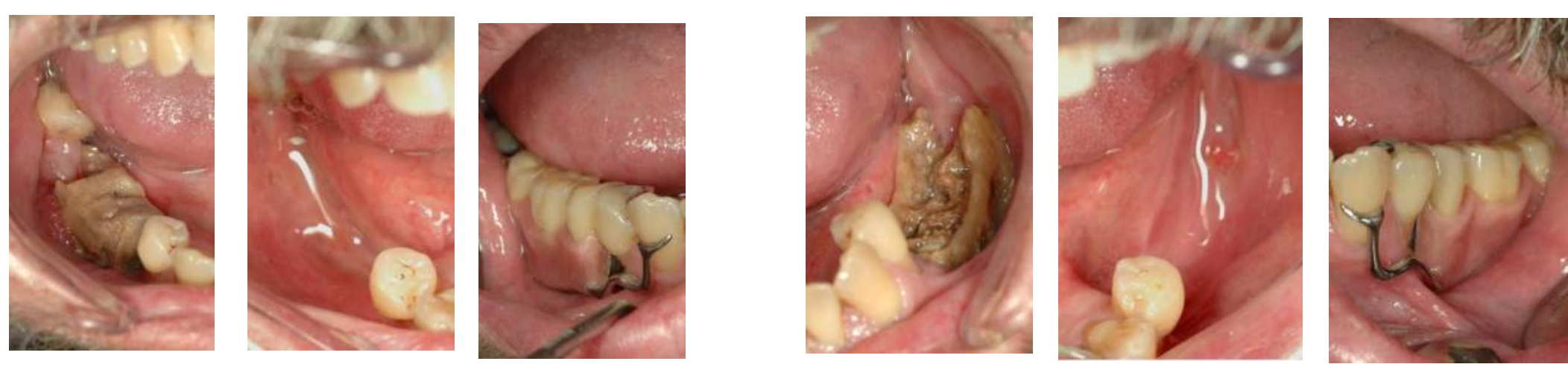

Example Case 2: Maxilla exposure
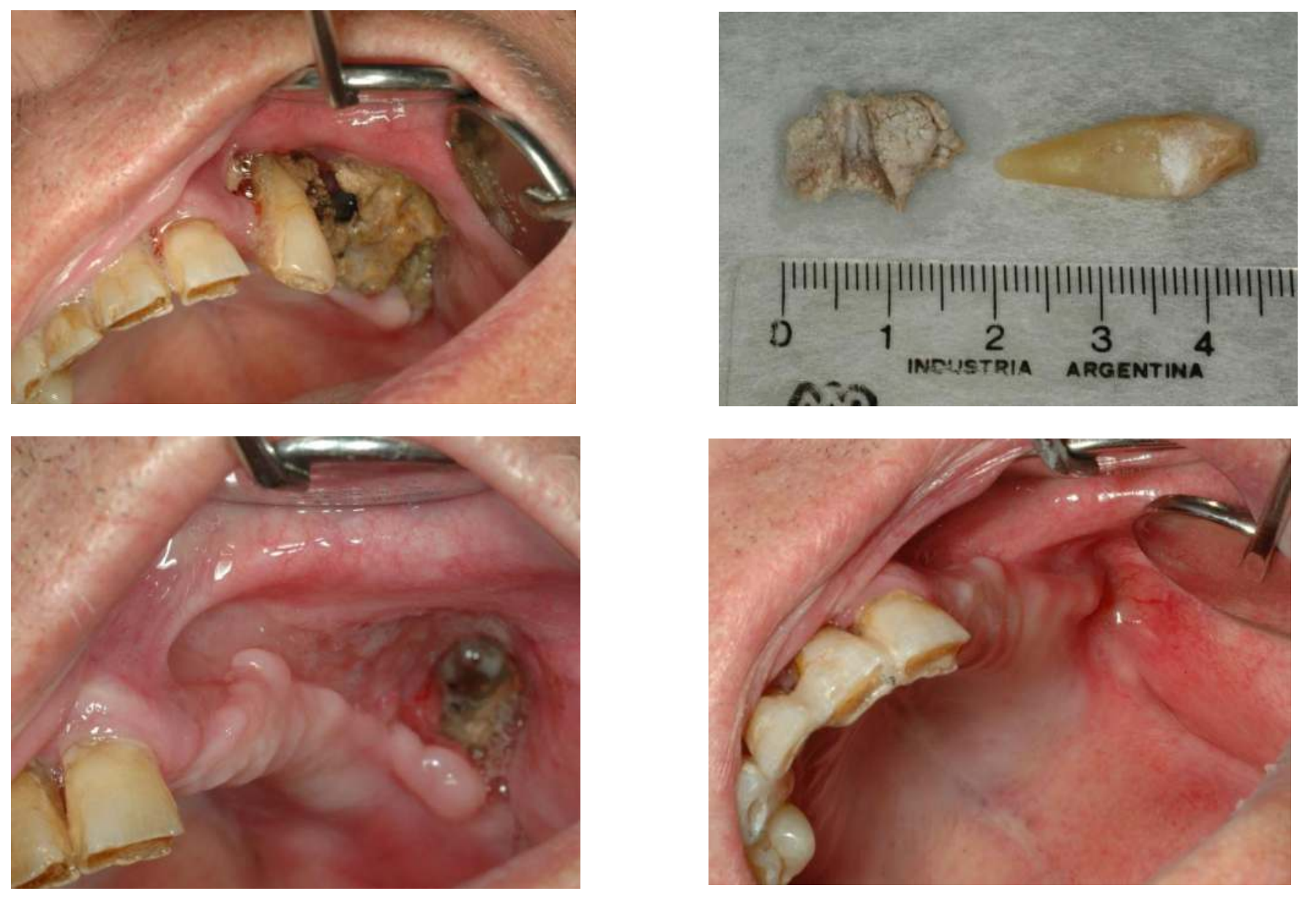

Example Case 3: Mandible exposure. Resolution and implants placement 7 years later
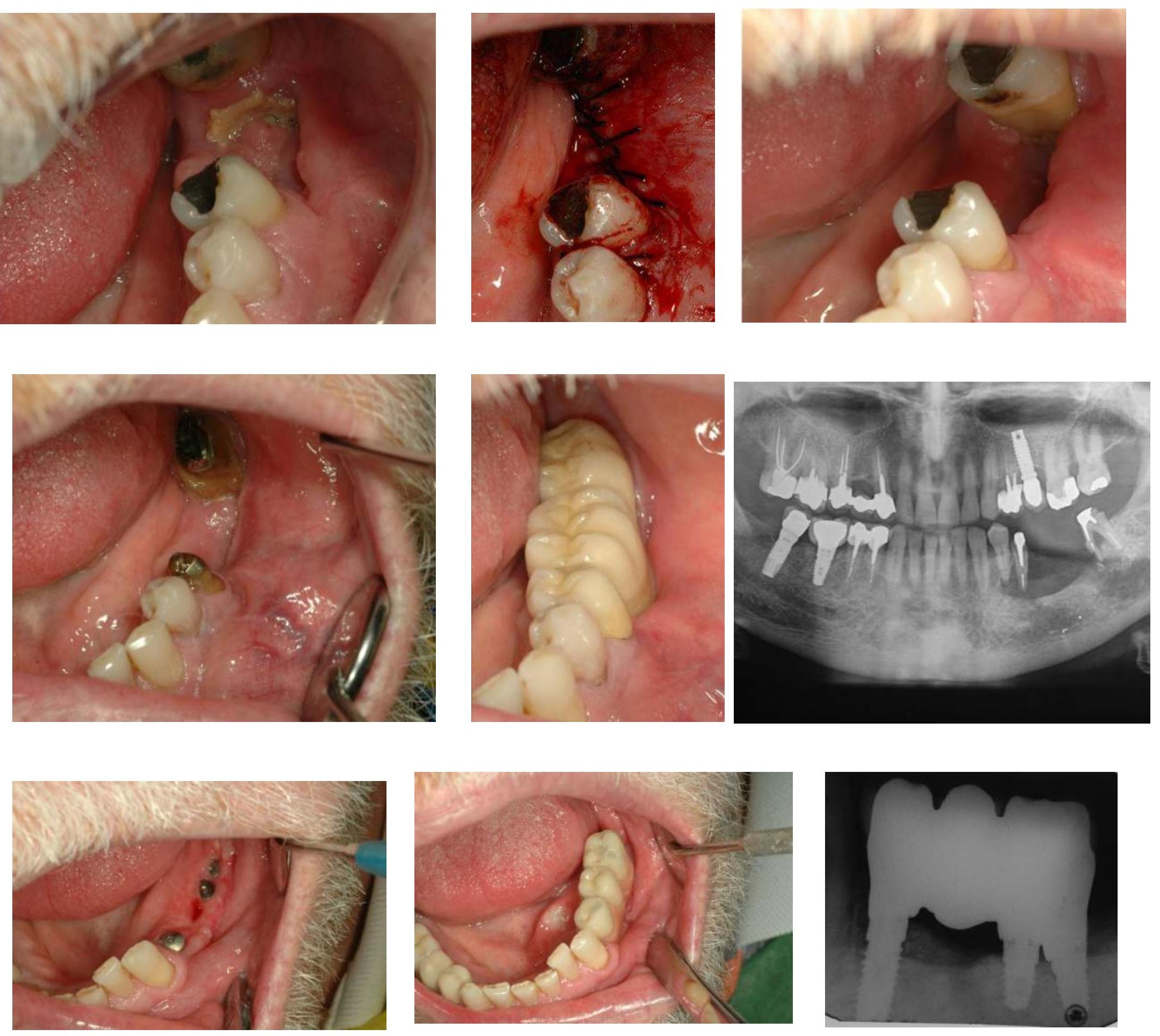

Results

In all cases, after the procedure was done, the soft tissue healed after around one month.

No new bone exposure were observed.

31 cases received no more treatment, 14 cases were delivered removable prosthesis, and in one case, dental implants were placed 7 years after the BRONJ treatment, and they were successful after 3 attempts. The last case was performed due to patient request and based in favorable values of Serum C-terminal telopeptide (CTX) test.

\section{Conclusions}

The latest literature, and also this study, supports the concept that BRONJ may be successfully treated. The approach described here, especially regarding bone exposure management, could minimize necessary surgical corrections upon removal of the sequestrum.

The resolution of these cases does not ensure that every case can be resolved in the same way or with the same results, but shows us that there is a real possibility to treat this pathology with success. Each case must be evaluated individually and primary approaches must always be conservative and focused on prevention. 